\title{
Bilateral Negotiation Decisions with Uncertain Dynamic Outside Options *
}

\author{
Cuihong Li \\ Tepper School of Business \\ Carnegie Mellon University \\ 5000 Forbes Avenue \\ Pittsburgh, PA 15213 \\ cuihong@andrew.cmu.edu
}

\author{
Joseph Giampapa \\ Robotics Institute \\ Carnegie Mellon University \\ 5000 Forbes Avenue \\ Pittsburgh, PA 15213 \\ garof@cs.cmu.edu
}

\author{
Katia Sycara \\ Robotics Institute \\ Carnegie Mellon University \\ 5000 Forbes Avenue \\ Pittsburgh, PA 15213 \\ katia@cs.cmu.edu
}

\begin{abstract}
Negotiation is an important phase of e-contracting. E-contracting requires a proper negotiation model to effectively support negotiation decisions or automate the negotiation process. When an entity negotiates with a potential contractor, there may be some alternatives that exist simultaneously with the potential contractor, and/or some may present themselves in the future. We present a model for bilateral contract negotiations that considers the uncertain and dynamic outside options. Outside options affect the negotiation strategies via their impact on the reservation price. The model is composed of three modules, single-threaded negotiations, synchronized multi-threaded negotiations, and dynamic multi-threaded negotiations. These three models embody increased sophistication and complexity. The single-threaded negotiation model provides negotiation strategies without specifically considering outside options. The model of synchronized multi-threaded negotiations builds on the single-threaded negotiation model and considers the presence of concurrently existing outside options. The model of dynamic multi-threaded negotiations expands the synchronized multi-threaded model by considering the uncertain outside options that may come dynamically in the future. Experimental analysis is provided to characterize the impact of outside options on the negotiation strategy and performances.
\end{abstract}

* This work was supported in part by the Navy Personnel Research, Studies and Technology (NPRST) under Navy contract number N6610-98-D-9501 and by a subcontract with Resource Consultants, Inc (RCI), subcontract number 03-177-7048/086.

\section{Introduction}

Negotiation is an important phase of econtracting [8, 13], as a fundamental and effective way for the participants to reach a mutually acceptable agreement on the contract. E-contracting requires a proper quantitative decision model of contract negotiations to provide formal effective support to either human or computer programs in the negotiation process [2]. Usually when an entity negotiates with a potential contractor, she can have other alternatives that exist simultaneously with the potential contractor, or may present themselves in the future while the negotiator is bargaining. For example, a company that considers outsourcing the work of IT maintenance may negotiate with IBM and at the same time also ask HP to make competitive offers. During the process of negotiation, some other smaller IT service providers may also show interest and get into the list of potential providers. The alternatives to one candidate are called outside options, and they contribute to the environment of the negotiation with the candidate. In the above example HP and other providers are outside options of IBM. Accepting a proposal from IBM means refusing all outside options. On the other hand the company may leave the negotiation with IBM (called "opt-out") without reaching an agreement based on the expectation of reaching a more favorable agreement with HP or the other potential providers. We call the negotiation between two parties a negotiation thread. For the convenience of presentation we will generally call the two parties in a negotiation thread a buyer and a seller, and the model is presented from a buyer's perspective. The subject of the exchange to contract upon is called an item. 
Outside options can execute concurrently with a negotiation thread, or present sequentially in the future. A concurrently executing outside option is a negotiation thread that the negotiator executes in parallel with another thread. A sequentially available outside option is an uncertain exchange opportunity that may come in the future with both the other party (and hence the item/service to contract) and the timing of presence uncertain.

Outside options impact the input to a negotiation decision model as a part of the environment. The existence of outside options changes the utility that the buyer expects from the current negotiation, and hence the agreement that is acceptable for the buyer in the current negotiation. We claim that outside options affect the negotiation strategies via their impact on the reservation price. The reservation price is the worst agreement that a negotiator can accept. For example, in the service outsourcing situation the reservation price of the customer company (buyer) is the highest price she is willing to pay for the service. The reservation price of the buyer depends on the value of the seller's service, and also on the availability of other providers. The reservation utility of the buyer in a negotiation is the utility of the worst acceptable agreement, which is reached at the reservation price. Therefore we have the following equation:

$$
\text { reservationprice }=\text { value }- \text { reservationutility } .
$$

If there are no outside options, the reservation utility is zero, and the reservation price held by the buyer is equal to the value of the item provided by the seller.

If there are outside options, the reservation utility in a negotiation is equal to the expected utility from outside options that are available now or later. The utility from outside options is measured on expectation because of the uncertainty of the availability and quality of outside options. We should remark that the claim is based on the assumption that a thread is non-resumable. In a resumable thread a negotiator can leave the negotiation table temporarily for discovering more information in other negotiation threads, and come back to resume the negotiation if necessary. In this work we assume that the negotiation threads are non-resumable. This assumption is not very restrictive because prior research on bargaining $[6,14]$ shows that having the option to suspend and resume a negotiation does not improve the utility of a negotiator in common bargaining situations.

Design of an effective negotiation strategy can be divided into two parts: the first is the design of a negotiation strategy given the reservation price and other inputs, the second is to calculate the reservation price based on the model of outside options. We call the model in the first part single-threaded negotiations.

The model of outside options can be built with two levels of complexity based on the two forms of availability of outside options. On the first level we can assume there are no outside options coming in the future. The outside options are those negotiation threads that concurrently exist with the thread under consideration. In other words, all negotiation threads are assumed to start at the same time. Therefore there is no uncertainty about outside options in terms of both the thread number and item values. We call this model of negotiations with only concurrently available outside options synchronized multi-threaded negotiations. On the second level we also consider the outside options that may come dynamically in the future. Hence the number of threads that the negotiator would be involved in is a random variable and changes with time. The item values in the future threads are also uncertain. We call this model with both concurrently and sequentially available outside options $d y$ namic multi-threaded negotiations. It builds on the synchronized multi-threaded model but introduces uncertainty on the threads. In both models of synchronized and dynamic multi-threaded negotiations the negotiation strategy in one thread can be derived from the single-threaded negotiation model, but the reservation price is calculated with the corresponding model of outside options. Figure 1 shows the relationship between these three negotiation models.

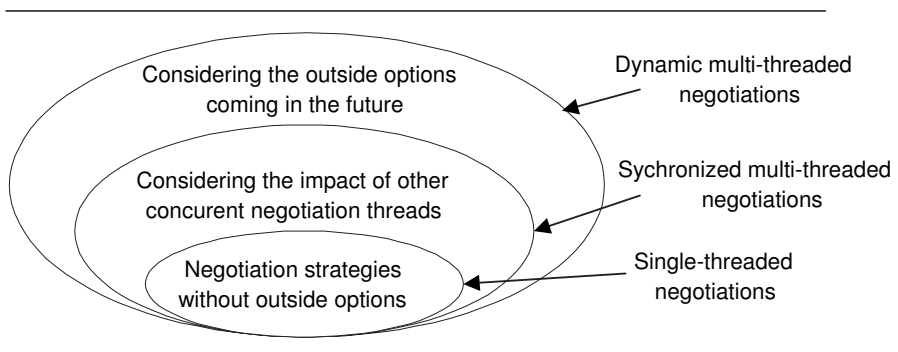

Figure 1. A nested view of the model

The rest of the paper is organized as follows: We review related literature in Section 2. Section 3 presents the specific model of each module. In Section 4 we provide experimental results. Section 5 concludes.

\section{Related work}

The research work on bilateral negotiations has been conducted in the fields of game theory, and artificial intelligence (AI). The research in game theory focuses on 
the outcome that satisfies given axioms, or the strategy equilibrium of agents, based on rigorous assumptions. Heuristic approaches are usually used in the complex situations for which game theoretic analysis is untractable. In Li, et al. [11] we provide an extensive research literature review on quantitative bilateral negotiation models in both fields of game theory and AI.

For a survey on negotiation models in the AI field please refer to Jennings et al. [9] and Gerding et al. [7]. Nguyen and Jennings [16] presents a heuristic model that enables an agent to participate in multiple, concurrent bi-lateral negotiations without considering the uncertain future arrivals. In game-theoretic research on bilateral negotiations most of the works that consider outside options assume that negotiators have complete information about each other's preferences $[3,21,4,14]$. Gantner [6] presents a bilateral negotiation model with incomplete information and an alternating-offers protocol. The outside option is modelled as a standard sequential search process. To simplify the analysis the paper assumes that there are only two types, high or low, for a negotiator. In this paper we consider a general situation in which the type space of a negotiator is continuous, and outside options are also negotiations that may happen simultaneously and dynamically.

\section{The model}

There are $T$ periods over the entire horizon for a buyer searching for a negotiation agreement to buy an item from a seller. Let a period be denoted by $t$, $t=0, \ldots, T-1$. A buyer needs to reach an agreement with a seller before period $T$. The potential sellers may come at different times unexpectedly with different reservation prices, and the buyer can negotiate with the sellers simultaneously. The number of threads in period $t$ is denoted by $n_{t}$, and the collection of threads in period $t$ is denoted by $D_{t}=\left\{d_{i}\right\}_{i=1}^{n_{t}}$. The seller in the thread $d_{i}$ is denoted by $s_{i}$. For simplicity we define the value of a seller as the value of the item provided by the seller. Let the value of the seller $s_{i}$ be $v_{i}$. If the buyer reaches an agreement with the seller $s_{i}$ at $x$, then the utility of the buyer is $v_{i}-x$.

The buyer wants to reach the lowest possible agreement with a seller by a negotiation with an alternatingoffers protocol. In an alternating-offers protocol the negotiators propose and respond alternatively, until one accepts an offer or quits the negotiation, or the negotiation deadline $T$ is reached. The actions at each step of a negotiator in an alternating-offers protocol include: accept, reject and propose an offer, quit. Both negotiation parties do not know the reservation price of each other. Assume the buyer has an estimation of the reservation price of a seller, and the estimation is characterized by a probability distribution $F(\cdot)$, where $F(x)$ denotes the probability that the reservation price of a seller is no greater than $x$. This probability distribution is called the prior belief of the buyer. A negotiation strategy specifies the action at each step conditional on the negotiation history ${ }^{1}$, and based on the reservation price and prior believes of the negotiators.

The reservation utility $O U_{i}$ in thread $d_{i}$ is equal to the expected utility from the outside options, which can be viewed together as a multi-threaded negotiation. Given the reservation utility $O U_{i}$, the reservation price $R_{i}$ of the buyer in thread $d_{i}$ can be calculated by $R_{i}=v_{i}-O U_{i}$. If the reservation price in a thread is known, the buyer can apply the single-threaded negotiation model to make the negotiation decisions in the thread.

Calculation of the expected utility from the outside options depends on the model on the outside options, and on the approach to estimate the expected utility from a multi-threaded negotiation. In a synchronized multi-threaded negotiation model the outside options at period $t$ for thread $d_{i}$ are the other concurrently existing negotiation threads $D_{t} \backslash d_{i}$. The synchronized model maps the current outside options to the reservation utility $O U_{i}\left(D_{t} \backslash d_{i}\right)$ of each thread $d_{i}, i=1, \ldots, n_{i}$. The dynamic multi-threaded negotiation model further considers the outside options that may come in the future at uncertain times with uncertain values, and can be viewed as a synchronized model with uncertain threads.

In the following sections $3.1,3.2$ and 3.3 these models are presented individually.

\subsection{Single-threaded negotiations}

To calculate the optimal negotiation strategy requires game theoretic analysis of the strategy equilibrium. This analysis is not tractable when both parties have incomplete information and the negotiation is based on an alternating-offers protocol [1]. In the AI field some effective heuristic negotiation strategies have been developed to provide formal decision models for automated negotiation agents. Among the generic single-issue quantitative models there are [5], [23], and [19], etc.. Since the focus of this paper is not in designing a single-threaded negotiation strategy, we adopt the time-dependent negotiation strategy that is developed in Faratin et al. [5], for its simplicity, to illustrate the integrative negotiation model with outside options.

1 The history of a negotiation at time $t$ is a sequence of the negotiators' actions before $t$. 
In the time-dependent approach, the proposal $x_{b}^{t}$ to be offered by a buyer and the value $x_{s}^{t}$ to be offered by a seller at time $t, t \in[0, T-1]$, are as follows:

$$
\begin{gathered}
x_{b}^{t}=\min _{b}+\alpha_{b}(t)\left(\max _{b}-\min _{b}\right), \\
x_{s}^{t}=\min _{s}+\left(1-\alpha_{s}(t)\right)\left(\max _{s}-\min _{s}\right),
\end{gathered}
$$

where $\max _{b}\left(\min _{s}\right)$ is the reservation price of the buyer (seller), $\min _{b}\left(\max _{s}\right)$ is the lower (upper) bound of a valid offer of the buyer (seller) (reasonably we can assume $\min _{b}=0$ ), and $\alpha_{i}(t)$ decides the pace of concession at time $t$. The buyer accepts an offer $x_{s}^{t}$ at time $t$ if it is not worse than the offer she would submit next time, i.e., $x_{b}^{t+1} \geq x_{s}^{t}$. Similarly the seller $s$ accepts an offer $x_{b}^{t}$ at time $t$ if $x_{s}^{t+1} \leq x_{b}^{t}$.

The time-dependent function $\alpha_{i}(t), i \in\{b, s\}$, can be defined by a family of polynomial functions ${ }^{2}$ :

$$
\alpha_{i}(t)=\left(\frac{t}{T}\right)^{\frac{1}{\beta}} .
$$

The constant $\beta>0$ determines the concession pace along with time, or the convexity degree of the curve of proposals. By varying $\beta$ a wide range of negotiation strategies can be characterized. With $\beta<1$ very small a negotiator takes a Boulware strategy [18], i.e., tends to maintain the offered value until the time is almost exhausted, then she concedes to the reservation price quickly. With $\beta>1$ very large a negotiator takes a Conceder strategy [17], i.e., goes to the reservation price rapidly and early. No matter what value $\beta$ takes, with a constant reservation price, the offer monotonically increases (decreases) with time for a buyer (seller) based on the time-dependent negotiation strategy.

\subsection{Synchronized multi-threaded negotia- tions}

In a synchronized multi-threaded negotiation process a negotiator participates in multiple bilateral negotiation threads with different, simultaneous negotiation opponents. The negotiator can reach an agreement in at most one of these threads, and is aware of all the threads at the beginning of the process. From one thread's perspective the other threads are outside options. The reservation utility that the negotiator should set in one thread is equal to the expected utility from all other threads. The other threads form a synchronized multi-threaded negotiation with one less thread than the original process.

A synchronized multi-threaded negotiation process is different from an auction in which the negotiator is

2 Alternatively we can also use the exponential function family, and define $\alpha_{i}(t)=e^{\left(1-\frac{t}{T}\right)^{\beta}}[5]$. the auctioneer and the negotiation opponents are the bidders, in that (1) in the former mechanism the communications between opponents and the negotiator are not synchronized as in an auction; (2) the messages between the negotiator and an opponents are not observed by other opponents, but in an open auction all bidders see the bids of other bidders and are informed of the provisional winner; and (3) in the negotiation mechanism (with an alternating-offers protocol) both parties can propose and respond, but in an auction only bidders propose and the auctioneer only responds. Despite the differences, both mechanisms drive competitions. The competition among bidders in an auction is realized via transparent and synchronized communications, and/or the winner selection mechanism. In a synchronized multi-threaded negotiation the negotiator achieves indirect competition among opponents since the negotiator sets a reservation price that is more aggressive than she would without outside options.

It is reasonable to assume that if any agreement is reached in a multi-threaded negotiation, the agreement is signed with the most competitive opponents among all opponents of the threads. For a buyer the seller $s_{i}$ in thread $d_{i}$ is more competitive than the sellers in other threads if $s_{i}$ can give more utility to the buyer, i.e., $y_{i}=v_{i}-r_{i}$ is greater than $y_{j}, d_{j} \in D \backslash d_{i}$, where $r_{i}$ is the reservation price the seller in thread $d_{i}$, and $D=\left\{d_{1}, \ldots, d_{N}\right\}$ is the collection of threads. The amount $y_{i}$ is the maximum utility that the buyer can achieve from the negotiation thread $d_{i}$. Let $G_{i}(y)$ denote the probability of the maximum utility in thread $d_{i}$ being less than $y$. Let $G^{1}(y)$ and $G^{2}(y)$ be the probability distribution of the highest and second highest maximum utility. The probability density functions of $G_{i}(y), G^{1}(y)$ and $G^{2}(y)$ are denoted by $g_{i}(y), g^{1}(y)$ and $g^{2}(y)$ respectively. These probabilities can be calculated by the following formulas:

$$
\begin{gathered}
G_{i}(y)=\operatorname{Pr}\left(v_{i}-r_{i} \leq y\right)=\operatorname{Pr}\left(r_{i} \geq v_{i}-y\right)=F\left(v_{i}-y\right), \\
G^{1}(y)=\prod_{d_{i} \in D} G_{i}(y), \\
G^{2}(y)=G^{1}(y)+\sum_{i=1}^{N}\left(1-G_{i}(y)\right) \prod_{d_{j} \in D \backslash d_{i}} G_{j}(y) .
\end{gathered}
$$

The corresponding probability density functions, or the derivatives of these (cumulative) probability distribution functions, are as follows:

$$
\begin{gathered}
g_{d}(y)=-f\left(v_{d}-y\right), g^{1}(y)=\sum_{d_{i} \in D} g_{i}(y) \prod_{d_{j} \in D \backslash d_{i}} G_{j}(y), \\
g^{2}(y)=g^{1}(y)-\sum_{i=1}^{N} g_{i}(y) \prod_{d_{j} \in D \backslash d_{i}} G_{j}(y)+\sum_{i=1}^{N}(1- \\
\left.G_{i}(y)\right)\left[\sum_{d_{j} \in D \backslash d_{i}} g_{j}(y) \prod_{d_{m} \in D \backslash\left\{d_{i}, d_{j}\right\}} G_{m}(y)\right] .
\end{gathered}
$$

We provide four heuristic approaches to estimate the expected utility $O U(D)$ from a multi-threaded negotiation composed by the threads $D$ : 
1. Conservative estimation: A synchronized multithreaded negotiation is approximated by an auction. The utility of the buyer is equal to the expected second highest maximum utility, as is the result in a reverse auction in which the buyer is the auctioneer and the opponents are the bidders [10]. The expected utility is calculated by

$$
O U=\int_{0}^{\bar{y}} y g^{2}(y) d y
$$

where $\bar{y}$ is the upper bound of the possible utility that the negotiator can achieve. If the lower bound of an acceptable price for a seller is $\underline{c}$, and the upper bound of a buyer's valuation is $\bar{v}$, then $\bar{y}=\bar{v}-\underline{c}$.

2. Medium estimation: A synchronized multithreaded negotiation is approximated by an English auction continued with a single-threaded negotiation between the buyer and the winning seller. Assume the single-threaded negotiation ends at the middle point between the buyer's and the winning seller's reservation price, if the buyer's reservation price is higher than the winning seller's ${ }^{3}$. In the continued single-threaded negotiation the reservation price of the buyer is equal to the last offer of the second most competitive seller in the auction, which brings a utility equal to the second highest maximum utility ${ }^{4}$. Then the expected utility is the average of the expected highest and second highest maximum utility.

$$
O U=\left(\int_{0}^{\bar{y}} y g^{2}(y) d y+\int_{0}^{\bar{y}} y g^{1}(y) d y\right) / 2
$$

In this estimation we assume an agreement can be reached as long as there is a zone of agreement, i.e., the buyer's reservation price is higher than a seller's. In the next approach of uniform approximation we further consider the probability that a negotiation may fail even if there is a zone of agreement, when negotiators do not know each other's reservation prices.

3. Uniform approximation: Previous research has established an optimal bargaining result between a buyer and a seller based on game theoretic analysis when both parties' reservation prices follow uniform distributions [15]. Based on this result, an agreement occurs if and only if the buyer's valuation exceeds the seller's cost by at least $1 / 4$, if both parties' reservation prices distribute uniformly on $[0,1]$. We can approximate the probability distributions of negotiators' types by uniform distributions and apply this result to calculate the probability of reaching an agreement. In the heuristic we assume an agreement cannot be reached in the continued single-threaded negotiation between the buyer

3 If the buyer's reservation price is lower than the seller's, there is no "zone of agreement" and the negotiation will fail.

4 In an English auction the optimal strategy of a bidder (seller) is to bid down to the true cost. and the winning seller if the maximum utility of the winning seller is less than a quarter of the highest possible utility $\bar{y}$. In this case the buyer achieves the second highest maximum utility, which is the reservation utility of the buyer in the continued single-threaded negotiation. If an agreement is reached in the singlethreaded negotiation, it is reasonable to assume that it is at the middle point between both parties' reservation prices. Therefore in this case the buyer achieves the medium of the highest and the second highest maximum utility.

$$
\begin{aligned}
O U= & \frac{\int_{0}^{\bar{y}} y g^{2}(y) d y+\int_{0}^{\bar{y}} y g^{1}(y) d y}{2} \int_{\bar{y} / 4}^{\bar{y}} g^{1}(y) d y+ \\
& \int_{0}^{\bar{y}} y g^{2}(y) d y\left(1-\int_{\bar{y} / 4}^{\bar{y}} g^{1}(y) d y\right) .
\end{aligned}
$$

4. Learning: Learn the probability of reaching an agreement and the distribution of agreements based on the previous negotiations [22]. The result of learning is represented by $x(v, c)$, the expected outcome of the negotiation when the buyer's and seller's reservation prices are $v$ and $c$ respectively. If the seller $s_{i}$ in the thread $d_{i}$ is the winning seller, then the probability distribution of her reservation price is $F(c) \prod_{d_{j} \in D \backslash d_{i}}(1-$ $\left.F\left(v_{j}-v_{i}+c\right)\right)$, where the product is the probability that no other thread $d_{j}$ has the maximum utility $v_{j}-c_{j}$ greater than the maximum utility $v_{i}-c_{i}$ in thread $d_{i}$. Given the expected outcome $x(v, c)$, the expected utility from a multi-threaded negotiation can be approximated by

$O U=\sum_{d_{i} \in D} \int_{\underline{c}}^{\bar{c}}\left(v_{d}-x\left(v_{i}, c\right)\right) \prod_{d_{j} \in D \backslash d_{i}}\left(1-F\left(v_{j}-v_{i}+c\right)\right) d F(c)$

If negotiators use the time-dependent strategy and the parameter $\beta$ is chosen randomly with the mean equal to 1 , then we expect negotiators to concede constantly on average. Then the result of learning is expected to be close to the result of negotiation when $\beta=1$ for both negotiators:

$$
x(v, c)= \begin{cases}\frac{v}{1+v-c} & \text { if } v \geq c \\ \emptyset & \text { otherwise }\end{cases}
$$

assuming the upper bound of an offer is 1 and the lower bound is $0^{5}$.

\subsection{Dynamic multi-threaded negotiations}

During a searching process negotiation opponents can be discovered sequentially and new negotiations

5 With $\beta=1$, the proposal by the buyer at time $t$ is $x_{b}^{t}=v t / T$, and by the seller is $x_{s}^{t}=1-t(1-c) / T \cdot x_{s}^{t}=x_{b}^{t}=v /(1+v-c)$ when $t=T /(1+v-c)$. 
are launched dynamically. For an ongoing negotiation thread the outside options not only include the other simultaneous negotiation threads, but also the threads that may be launched in the future. If a negotiator knows the number of outside options that will come, and the value of the opponent in each outside option, then the negotiator can apply the synchronized multithreaded negotiation model to calculate the appropriate reservation price in each thread. But usually a negotiator is not sure about the arrival of, and the opponents' values in, future outside options. The reservation utility of a thread is the expected utility of a multi-threaded negotiation - including other simultaneous threads and threads launched in the future - with a stochastic thread number and uncertain opponents.

Following a usual way of modelling uncertain arrival, we assume the arrival of outside options follows a Poisson process [12]. In each period there is probability $p$ that the negotiator finds an alternative and launches a negotiation thread. The granularity of each period is small enough so that the probability that there are more than one arrival in one period is zero. In a Poisson process the number of arrivals $\eta(\tau, p)$ during an interval with length $\tau$ follows a Poisson distribution, $P_{p, \tau}(n)=\operatorname{Pr}(\eta(\tau, p)=n)=e^{-p \tau} \frac{(p \tau)^{n}}{n !}[20]$. Denote by $\Phi(y)=\operatorname{Pr}(v \leq y)$ the probability that an opponent's value is no greater than $y$. A negotiator knows the value of an opponent when the opponent is identified, but not the reservation price of the opponent.

The state $s_{t}$ of the system is defined as the number of past or existing threads $n_{t}{ }^{6}$, and the value of each opponent $v_{d}$ in the thread, $s_{t}=\left\{n_{t},\left\{v_{d}\right\}_{d=1}^{n_{t}}\right\}$. Let $U_{t}\left(s_{t}\right)$ be the utility that the negotiator expects from the dynamic multi-threaded negotiation when she sees the system state $s_{t}$ at period $t$. Following Section 3.2 we can calculate $U\left(\left\{n,\left\{v_{d}\right\}_{d=1}^{n}\right\}\right)$, the expected utility from a synchronized multi-threaded negotiation with $n$ threads and the opponent in thread $d$ valued $v_{d}$, $d=1, \ldots, n . U_{t}\left(s_{t}\right)$ is the expectation of $U\left(s_{T-1}\right)$ with respect to $s_{T-1}$, which depends on $s_{t}$ :

$$
\begin{gathered}
U_{t}\left(s_{t}\right)=E_{\eta}\left[E_{\left\{v_{d}\right\}_{d=n_{t}+1}^{n_{t}+\eta}}\left[U\left(\left\{n_{t}+\eta,\left\{v_{d}\right\}_{d=1}^{n_{t}} \cup\left\{v_{d}\right\}_{d=n_{t}+1}^{n_{t}+\eta}\right\}\right]\right]\right. \\
U_{T-1}\left(s_{T-1}\right)=U\left(s_{T-1}\right) .
\end{gathered}
$$

where $\eta$ follows a Poisson distribution $P_{p, T-t}(\cdot)$, and $v_{d}$ independently follows the identical distribution $\Phi(\cdot)$, $d=n_{t}+1, \ldots, n_{t}+\eta$.

6 We count the past threads in the state because they affect the probability distribution of the maximum utilities of the existing threads. The threads that have survived generally have higher maximum utilities than the threads that have ended earlier.
To set the reservation price of a thread, the negotiator only needs to calculate the expected utility of the multi-threaded negotiation which does not include that thread, given the period and the state. Because the state of a dynamic multi-threaded negotiation changes from period to period, the reservation price of a thread may also changes with time.

The computation of the expected utility following Equation 4 because the number of states is exponential with respect to the number of opponent's values. If there are at most $N$ threads and for each opponent there are $M$ possible values, then the number of possible states will be $N^{M}$. To simplify the computation we can approximate the result by having the opponent value instances replaced by the expected value $\bar{v}$, i.e.,

$$
U_{t}\left(s_{t}\right)=E_{\eta}\left[U\left(\left\{n_{t}+t,\left\{v_{d}\right\}_{d=1}^{n_{t}} \cup\{\bar{v}\}_{d=n_{t}+1}^{n_{t}+\eta}\right\}\right]\right] .
$$

The compromise due to this simplification is not significant if the expected utility of a synchronized thread is or can be approximated by a linear function of the opponents' values.

\section{Experiments}

In Section 4.1 we show how the reservation utility of a negotiation thread evolves with time and the change of outside options in the synchronized and dynamic multi-threaded negotiation models. We then show the impact of outside options on the negotiation strategy by showing the offer curves adjusted by the reservation prices, compared with the original basic offer curve without considering outside options. In Section 4.2 we compare the average utility of a negotiator when she (1) does not consider outside options, (2) when she only considers concurrent outside options, i.e., the synchronized multi-threaded negotiation model, and (3) when she considers both concurrent outside options and future arrivals, i.e., the dynamic multi-threaded negotiation model.

In the experiments the negotiation deadline $T=20$. The buyer believes the reservation price of a seller follows a uniform distribution on the interval $[0,1]$. The value of a seller's item is also uniformly distributed on $[0,1]$. The probability that a new seller arrives in a period is $p$, and $p$ takes the values $\{0.05,0.10,0.15,0.20,0.25\}$. The parameter $\beta$ in the time-dependent strategy of a negotiator is chosen randomly so that with even probability a negotiator in a thread is a Conceder $(\beta>1)$ or a Boulware $(\beta<1)$. If a negotiator is a Conceder, $\beta^{-1}$ follows a uniform distribution on $[0,1]$. If a negotiator is a Boulware, $\beta$ is a random variable with a uniform distribution on $[0,1]$. For each arrival probability, we 
repeat the experiment 100 times and the average utility of the buyer is calculated. The expected utility of a dynamic multi-threaded negotiation process was calculated with the approximation formula, Equation 5 (Section 3.3).

\subsection{Reservation utilities and offer curves}

We illustrate the impact of outside options on the negotiation strategy by a specific example. In this example $p=0.2$ and $\beta=1.262727^{7}$. The values and arrival times of outside options in the instance are illustrated in Figure 2.

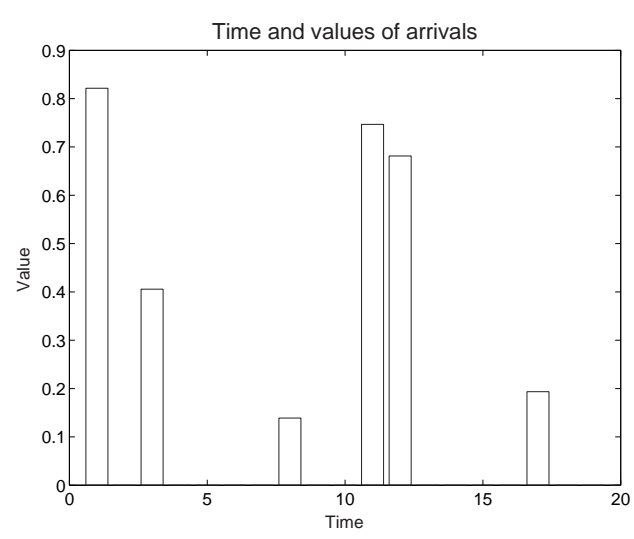

Figure 2. Time and values of arrivals

The offer curves in the first thread calculated based on different estimation approaches and outside option models are shown in Figure 3. The model noted by "Single" is the model without considering outside options. When the buyer does not consider outside options the offer increases with time as the buyer constantly concedes (with changing pace). But with a synchronized or dynamic model the buyer may proceed, i.e., decrease the offered price from the previous one, when a valuable new opponent arrives (e.g., at time 11). This is because the reservation utility of the buyer increases when she sees a new seller that offers a highvalue item. When there are no new arrivals, the buyer will concede in all these three models. The concession pace in the synchronized model is the same as in the single-threaded model, but it is greater in the dynamic model. This is because in the dynamic model the buyer expects fewer new arrivals and the reservation utility decreases with passing time. The offers without considering outside options are higher than the offers with

7 The multiple experiments with different $p$ and $\beta$ show the same pattern. considering only concurrent negotiation threads, which are again higher than the offers with additional considerations of outside options that may come in the future.
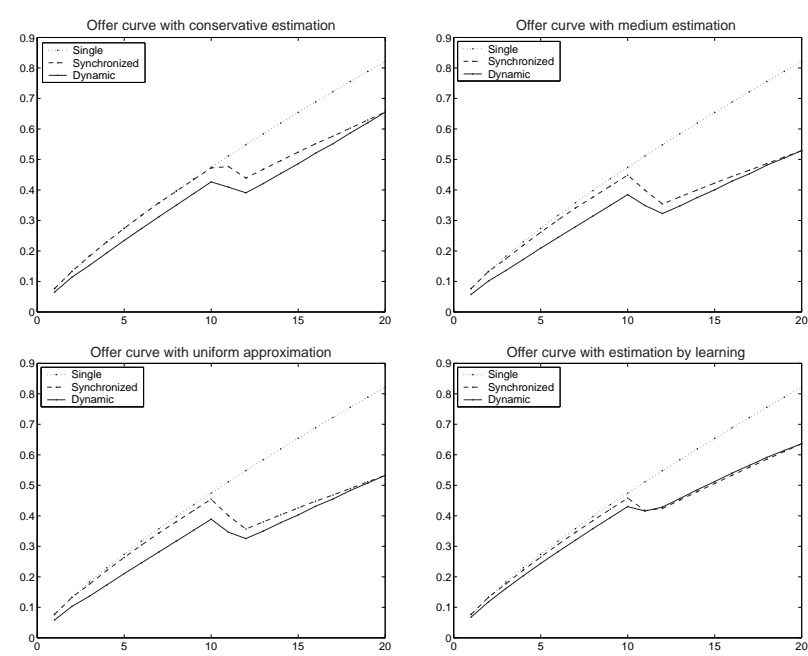

Figure 3. The offer curves

\subsection{Performance results}

Figure 4 is composed of four subplots. Each subplot shows the average utility as a function of the arrival probability based on one reservation utility estimation approach, and with different outside option models. The figure implies that for all estimation approaches and outside option models, the average utility increases with the arrival probability. This is intuitive and should be true for a reasonable negotiation strategy. A higher arrival probability implies more options on expectation and should result in better outcome for the negotiator. Figure 4 also shows that the average utility based on the dynamic model is higher than the one based on the synchronized model, which again brings higher average utility than the single-threaded model in which no outside option is considered. This verifies the effectiveness of the solution framework and the heuristic approaches we have proposed.

\section{Conclusion}

In this paper we provide an integrative solution for the contract negotiation decision problem when negotiators face uncertain and dynamic outside options. We do not claim that the heuristics we provide in this report are complete. Rather they reflect solutions that 

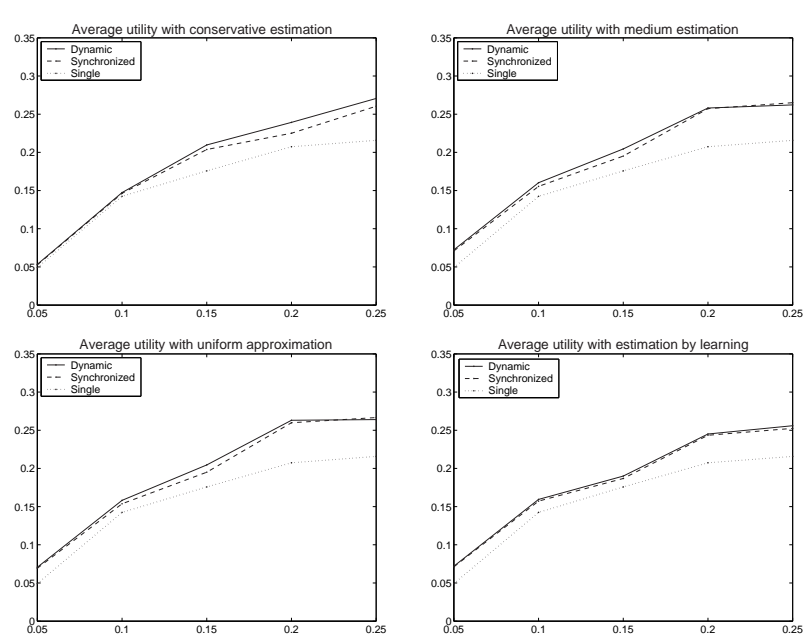

Figure 4 . The average utilities with varying arrival probability

have been proven useful or plausible. Other negotiation strategies and approaches to estimate the utility from a multi-threaded negotiation can be plugged in the solution framework, depending on the assumptions and requirements of the underlying application. These different models can construct a library of decision functions to support the decision of negotiation agents in different environments.

\section{References}

[1] L. Ausubel, P. Cramton, and R. Deneckere. Bargaining with incomplete information, volume 3, chapter 50. Elsevier Science, 2002. Robert J. Aumann and Sergiu Hart, eds.

[2] M. Bichler, G. Kersten, and S. Streker. Towards a structured design of electronic negotiations. Group Decision and Negotiation, 12(4):311-335, 2003.

[3] K. Binmore, A. Shaked, and J. Sutton. An outside option experiment. Quarterly Journal of Economics, 104(4):753-770, 1989.

[4] K. Chatterjee and C. C. Lee. Bargaining and search with incomplete information about outside options. Games and Economic Behavior, 22:203-237, 1998.

[5] P. Faratin, C. Sierra, and N. R. Jennings. Negotiation decision functions for autonomous agents. International Journal of Robotics and Autonomous Systems, 24(3-4):159-182, 1998.

[6] A. Gantner. Bargaining, search and outside options. Technical report, Department of Economics, University of California, Santa Barbara, 2002.

[7] E. H. Gerding, D. van Bragt, and J. L. Poutré. Scientific approaches and techniques for negotiation: a game theoretic and artificial intelligence perspective. Technical Report SEN-R0005, CWI, 2000.

[8] F. Griffel, T. Tu, M. Münke, M. Merz, W. Lamersdorf, and M. M. da Silva. Electronic contract negotiation as an application niche for mobile agents. In Proceedings of the 1st International Workshop on Enterprise Distributed Object Computing, October 1997.

[9] N. R. Jennings, P. Faratin, A. R. Lomuscio, S. Parsons, C. Sierra, and M. Wooldridge. Automated negotiation: prospects, methods and challenges. Int. J. of Group Decision and Negotiation, 10(2):199-215, 2001.

[10] V. Krishna. Auction Theory. Academic Press, 2002.

[11] C. Li, J. Giampapa, and K. Sycara. A review of research literature on bilateral negotiations. Technical Report CMU-RI-TR-03-41, Carnegie Mellon University, 2003.

[12] S. Lippman and J. McCall. The economics of uncertainty: Selected topics and probabilistic methods, volume I, chapter 6, In K.J. Arrow and M. Intriligator, editors. North-Holland Publishing Company, Amsterdam, 1981.

[13] M. Merz, F. Griffel, M. Boger, H. Weinreich, and W. Lamersdorf. Electronic contracting with cosmos how to establish, negotiate and execute electronic contracts on the internet. In Proceedings of the 2nd International Workshop on Enterprise Distributed Object Computing, November 1998.

[14] A. Muthoo. On the strategic role of outside options in bilateral bargaining. Operations Research, 43(2):292-297, 1995.

[15] R. B. Myerson and M. A. Satterthwaite. Efficient mechanisms for bilateral trading. Journal of Economic Theory, 29:265-281, 1983.

[16] T. D. Nguyen and N. Jennings. A heuristic model for concurrent bi-lateral negotiations in incomplete information settings. In Proceedings of International Joint Conferences on Artificial Intelligence, pages 1467-1469, Mexico, 2003.

[17] D. G. Pruitt. Negotiation Behavior. Academic Press, 1981.

[18] H. Raiffa. The Art and Science of Negotiation. Harvard University Press, 1982.

[19] J. Rosenschein and G. Zlotkin. Rules of Encounter. MIT Press, 1994.

[20] S. M. Ross. Introduction to Probability Models. Academic Press, 2000.

[21] A. Rubinstein. Perfect equilibrium in a bargaining model. Econometrica, 50(1):97-109, January 1982.

[22] K. Sycara. Machine learning for intelligent support of conflict resolution. Decision Support Systems, 10:121$136,1993$.

[23] D. D. Zeng and K. Sycara. Bayesian learning in negotiation. International Journal of Human-Computer Studies, 48:125-141, 1998. 